\title{
Gravure Printed Flexible Surface Enhanced Raman Spectroscopy (SERS) Substrate for Detection of 2,4-Dinitrotoluene (DNT) Vapor
}

\author{
Sepehr Emamian*, Ali Eshkeiti, Binu Baby Narakathu, Sai Guruva Reddy \\ Avuthu and Massood Z. Atashbar \\ Department of Electrical and Computer Engineering \\ Western Michigan University \\ Michigan-49008, USA
}

Corresponding authors:

*Email: sepehr.emamian@wmich.edu

Address: 4601 Campus Drive, Western Michigan University, Department of Electrical and Computer Engineering

Kalamazoo, Michigan - 49008

Tel: +1 2693701788 


\begin{abstract}
An efficient surface enhanced Raman spectroscopy (SERS) substrate was successfully fabricated by gravure printing a thin film of silver nanoparticle (Ag NP) ink, with average particle size of $150 \mathrm{~nm}$, on flexible polyethylene terephthalate (PET) sheet. The feasibility of the printed SERS substrate for detecting explosive organic compounds such as 2,4-dinitrotoluene (DNT), in vapor phase, was investigated. The SERS based response of the printed substrate demonstrated an enhancement factor of four in the intensity of the Raman signal of DNT vapor when compared to target molecules adsorbed on bare PET. An $85 \%$ decrease in the intensity of the Raman spectrum was also observed as the temperature increased from $25{ }^{\circ} \mathrm{C}$ to $65{ }^{\circ} \mathrm{C}$. The results obtained show the efficiency of the gravure printed SERS substrate to be used in applications for the detection of explosive organic compounds.
\end{abstract}

Keywords: Gravure Printing, SERS, PET, Silver Nanoparticles (Ag NP), 2,4-dinitrotoluene (DNT). 


\section{Introduction}

Detection of explosive compounds such as 2,4,6-trinitrotoluene (TNT) and 2,4dinitrotoluene (DNT) have been continuously researched throughout the history of the manmade wars $[1,2]$. Physical probing based methods, typically used to clear landmines, are often labor intensive and time consuming. Moreover, these methods often confront problems due to other metal debris in the minefield, thereby resulting in false positives. The detection of the vapors of the explosive compounds is thus a better solution to overcome the drawbacks associated with physical probing methods. DNT is known to be more concentrated in the vapor phase, when compared to TNT [3]. Therefore, the development of new methods that can be employed for the detection of explosive organic compounds in vapor phase is important.

Various sensing techniques such as surface acoustic wave (SAW) sensors [4-6], luminescence based sensors [7-9], refractive index (RI) based optical sensors [10-12], cantilever sensors [13,14], quartz crystal microbalance (QCM) sensors [15,16], electrochemical sensors [17-20] and optofluidic ring resonator sensors [21] and have been used for the detection of DNT vapors. However, these methods have some disadvantages such as low sensitivity, low detection specificity and need for elevated working temperatures. Raman spectroscopy (RS), which is a rapid, sensitive and low temperature method of detection, has also been used for the detection of DNT vapors [22]. RS has been proven to be a reliable technique for the detection of various compounds due to its non-destructive nature and ability to provide signature vibrational fingerprints of target molecules.

Raman effect, in RS, is based on the principle of excitation and inelastic scattering of photons caused by incident light striking a surface [23]. Typically, the detected signals in RS are weak because the number of photons scattered by these excitations are not enough, thereby 
resulting in lower optical efficiencies. Studies by Jeanmaire and Van Duyne [24] and Fleischmann et al. [25] and have focused on enhancing the Raman effect. It was demonstrated that nanoscale metallic surfaces such as nano-pillars [26] and nano-domes [27] can enhance the Raman scattering and this phenomenon is known as surface enhancement Raman spectroscopy (SERS). The effect of incident excitation light, on the surface of a metal, causes free electrons to resonate thereby inducing electromagnetic fields. The absorbed target molecules on the metal surface will therefore be exposed by more intense electromagnetic fields which increases the number of scattered photons [28]. The deposited metal NP on the substrate in the molecular scale is not uniform and this reasons diverse electromagnetic field on the surface focused around the area of NP. High enhancement could be attained at some points which are called hot spots that are shown in Fig. 1. [29]. The use of SERS is thus a promising solution for the rapid, highly sensitive and selective detection of explosive organic compounds.

Several methods such as chemical bath deposition [30], anodic alumina oxide membrane decorated [31], wet chemical fabrication [32], physical vapor deposition [33], and combination of top-down photolithography and hydrothermal growth [34] have been used for the fabrication of SERS substrates. One of the most popular approaches for fabricating SERS substrates is the deposition of metallic nanoparticles (NP), such as Silver (Ag) [35] and gold (Au) [36], close enough to form "hot spots". Different techniques such as mechanical deformation, lithography, chemical vapor deposition (CVD), electroplating and oblique angle deposition have been used to deposit Ag and Au NPs on various substrates [37-41]. The drawbacks associated with these deposition methods are their complexity, excessive time consumption and expensive procedures. The use of traditional printing methods such as inkjet and gravure printing, which are typically cost efficient and requires low working temperatures, has shown to overcome these drawbacks 
for the fabrication of SERS substrates $[42,43]$. However, there are no reports on the use of gravure printing of flexible SERS substrates for the detection of explosive organic compounds such as DNT in vapor phase.

The gravure printing process includes an image carrier which is the gravure cylinder, an ink fountain, doctor blade and an impression cylinder as shown in Fig. 2. The gravure cylinder, made of copper coated steel, has small recessed cells that are engraved onto its surface to form the desired image. These cells can be electromechanically engraved, electrochemically etched or laser engraved. The image carrying cells hold the ink when the cylinder is submerged into the ink fountain. The excess ink is wiped off by a doctor blade from the surface of the cylinder. The ink in the cells is then impressed onto the substrate by an impression cylinder which is made of rubber [44].

In this work, gravure printing has been employed to deposit a thin film of Ag NP ink on flexible polyethylene terephthalate (PET) sheet to be used as a SERS substrate. The capability of the printed substrate for detecting explosive organic compounds such as DNT, in vapor phase, was investigated. The effect of varying temperatures on the intensity of Raman spectrum obtained for DNT vapor was also studied.

\section{Experimental}

\subsection{Materials, chemicals and sample preparation}

A transparent PET (Melinex ST 506) film from DuPont Teijin Films was used as the substrate. A conductive NP based Ag ink, with average particle size ranging of $150 \mathrm{~nm}$, from InkTec Inc. was used for the metallic surface of the SERS substrate. Ethanol and DNT (in powder form) was purchased from Sigma-Aldrich Chemical Company. A saturated solution of DNT was prepared by mixing with ethanol. 


\subsection{SERS Substrate Fabrication}

One layer of Ag NP ink was gravure printed on the PET substrate, using a laboratory gravure press (K-Printing Proofer) from Testing Machines Inc. The printed substrate consisted of a row of $1 \mathrm{~cm} \times 2 \mathrm{~cm}$ blocks. The printed Ag NP was cured in a VWR 1320 temperature controlled oven for 20 minutes at $130{ }^{\circ} \mathrm{C}$. Figure 3 shows the gravure printed SERS substrate. A thickness of $900 \mathrm{~nm}$ was measured using a Bruker vertical scanning interferometer microscope (CounterGT) over an area of $125 \times 95 \mu \mathrm{m} 2$ for the printed Ag NP film, as shown in Fig. 4 (a) which depicts the 3D profile of the printed silver ink. The average roughness (Ra) was measured to be $85 \mathrm{~nm}$ (Fig. 4(b)) which is smaller than the wavelength of the incident excitation radiation. Research has shown that, for silver electrodes, maximum SERS signal are typically obtained when the surface roughness is in the range of $80-100 \mathrm{~nm}[45]$.

\subsection{Experimental Procedure}

The experiment setup for transferring the DNT vapor onto the printed SERS substrate is shown in Fig. 5. Initially, $1 \mathrm{ml}$ of the DNT saturated solution was deposited onto a wad of steel wool. The steel wool was then inserted into a heated copper pipe (inner diameter of $10 \mathrm{~mm}$ ). The temperature of the copper pipe was set to $80^{\circ} \mathrm{C}$ using a Staco variable autotransformer (3PN 1010) and a wraparound heating cord (Omegalux HTC 030). Nitrogen carrier gas was then blown through the heated pipe. The printed substrate was held at the outlet of the copper pipe for 60 seconds. The Raman spectrum of the SERS subtrate was then measured.

Figure 6 shows the experiment setup for the SERS measurement. A laser source in the near infrared region with a wavelength of $785 \mathrm{~nm}$ was used to excite the sample using a Raman probe (Inphotonics Inc.), with an integration time of $3 \mathrm{~s}$ at $300 \mathrm{~mW}$. A spectrometer (QE 65000 Ocean Optics, 780-1100 nm) was employed to obtain the Raman spectra through the collection 
fiber of the Raman probe. The Raman spectra were then analyzed using Spectra Suite software (Ocean Optics) for the Raman signature spectra of the target molecules.

\section{Results and discussion}

\subsection{SERS Response to DNT}

Initially, the Raman spectra of DNT solution and vapor on bare PET were measured as reference signals. Then, the Raman spectra of DNT solution and vapor on the printed SERS substrate were obtained. Figure 7 shows the comparison of the Raman spectra of DNT solution (Fig. 7(a)) and vapor (Fig. 7(b)) on the printed SERS substrate as well as the spectra on bare

PET. The Raman spectrum yielded a strong peak at $1350.13 \mathrm{~cm}^{-1}$, which corresponds to $\mathrm{NO}_{2}$ stretching modes in the DNT molecule and can be used as a fingerprint for the detection of DNT [46].

The enhancement factor (EF), which is defined as a comparison between the intensities of the Raman peaks obtained with and without the use of metallic NPs, under similar test conditions, was calculated. An EF of three and four for the peaks at $1350.13 \mathrm{~cm}^{-1}$ was obtained for the SERS based response of the printed SERS substrate towards DNT solution and vapor, respectively, when compared to target molecules adsorbed on bare PET. The obtained enhanced SERS response can be considered as the result of intense electromagnetic field caused by the hotspots created by the agglomeration of the printed nanoparticles [47]. The distance of the target molecule from the NPs plays an important role in the intensity of Raman spectrum. Placement of the analyte molecules within these regions causes SERS enhancement. In this phenomenon, it is important to obtain an accurate control over various parameters such as shape, size, and crystallinity of the NPs, as well as the distance between the NPs. In other words, the intensity of the electromagnetic field depends on size of "nanogaps" between the printed 
nanoparticles which is directly related to the nanoparticle dimension. Decreasing the size of these nanogaps can be achieved by decreasing the size of nanoparticles which in turn increases the intensity of the Raman signals [48].

The results obtained show the efficiency of the gravure printed SERS substrate to be used in applications for the detection of DNT, which is known as the most common nitro-aromatic compound in buried landmines and other explosives [3]. The response of the printed SERS substrate also shows that it can be employed for the detection of explosive organic compounds in both aqueous and gaseous states. Therefore, the printed SERS substrate is bound to have substantial effects in medical detection, biological and chemical warfare detection, as well as several security applications.

\subsection{Effect of Temperature on SERS Response}

In order to investigate the effect of temperature on the intensity of the Raman spectrum, the printed SERS substrate that was exposed to DNT vapors were heated from $25^{\circ} \mathrm{C}$ to $65^{\circ} \mathrm{C}$, in steps of $10{ }^{\circ} \mathrm{C}$ using a hot plate. Figure 8 shows the response of the printed SERS substrate towards varying temperatures. It was observed that the intensity of the Raman spectrum decreased by $85 \%$ as the temperature was increased from $25^{\circ} \mathrm{C}$ to $65^{\circ} \mathrm{C}$. The effect of temperature on the intensity of the Raman spectra has been studied by Xie et al. [49] in which the decrease in the intensity of Raman spectrum with increasing temperature is attributed to an increase in the optical absorbance of the target molecule at the frequencies involved in the Raman process. The results obtained show that the vibration of the surface plasmon changes with respect to the temperature, which in turn affects the incident radiation frequency dependence of the SERS response. In another words, the vibrational energy of the molecules will increase as the temperature increases thereby reducing the bonding force between the molecules; thus 
decreasing the intensity of the Raman spectrum [50]. These responses also demonstrate that the number of hotspots can be optimized by controlling temperature to obtain maximum coupling between adjacent particles and matching the plasmon resonance wavelength to the laser excitation wavelength.

\subsection{Effect of Bending on SERS Response}

The effect of bending, on the intensity of the Raman spectrum, was investigated by flexing the SERS substrate to different radius of curvatures $(3.4 \mathrm{~cm}, 2.1 \mathrm{~cm}$ and $1.6 \mathrm{~cm})$, while exposing it to DNT vapors. Figure 9 shows the response of the printed SERS substrate towards varying bending angles. It was observed that the intensity of the Raman spectrum increased by $100 \%, 200 \%$, and $300 \%$ when compared to the Raman intensity of the flat SERS substrate, for the peak at $1350.13 \mathrm{~cm}^{-1}$. While bending the NPs come closer as a result number of hotspots increases. This creates a stronger electromagnetic field which can be attributed to the enhancement in the intensity of the Raman spectrum. These results demonstrate that bending the substrate can be an advantage in detection of the target molecules.

\section{Conclusion}

A SERS substrate was successfully designed and fabricated by gravure printing Ag NP ink on flexible PET. The feasibility of the printed substrate to be used as a SERS substrate for the detection of explosive materials such as DNT was demonstrated. An enhancement factor of three and four for the peaks at $1350.13 \mathrm{~cm}^{-1}$ was obtained for the SERS based response of the printed SERS substrate towards DNT solution and vapor, respectively, when compared to target molecules adsorbed on bare PET. The effect of temperature on the intensity of Raman spectrum was also examined. An $85 \%$ decrease in the Raman intensity was observed at $65{ }^{\circ} \mathrm{C}$ when compared with the intensity of Raman spectrum at $25^{\circ} \mathrm{C}$. The effect of bending on the SERS 
response was also investigated. The intensity of the Raman spectrum was increased as the SERS substrate was bended by different radius of curvature. Further research is underway for the development of a gravure printed SERS substrate using different substrates such as paper to be used in hand-held SERS based systems for the detection of a wider range of bio/chemicals. Moreover, the fabricated SERS substrates have the promising potential to be used as a cost effective substitution in commercialized SERS detection applications.

\section{Acknowledgments}

This work has been partially supported by the U.S. Army Grant Nos.WS911QY-07-1 0003 and W911NF-09-C-0135. 


\section{References}

[1] S. Pramanik, C. Zheng, X. Zhang, T.J. Emge, J. Li, New microporous metal-organic framework demonstrating unique selectivity for detection of high explosives and aromatic compounds, J. Am. Chem. Soc. 133 (2011) 4153-4155.

[2] S.J. Toal, W.C. Trogler, Polymer sensors for nitroaromatic explosives detection, J. Mater. Chem. 16 (2006) 2871-2883.

[3] J.M. Sylvia, J.A. Janni, J.D. Klein, K.M. Spencer, Surface-enhanced Raman detection of 2,4dinitrotoluene impurity vapor as a marker to locate landmines, Anal. Chem. 72 (2000) 5834-5840.

[4] G. Kannan, A. Nimal, U. Mittal, R. Yadava, J. Kapoor, Adsorption studies of carbowax coated surface acoustic wave (SAW) sensor for 2,4-dinitro toluene (DNT) vapor detection, Sens. Actuator B-Chem. 101 (2004) 328-334.

[5] E.J. Houser, T.E. Mlsna, V.K. Nguyen, R. Chung, R.L. Mowery, R. Andrew McGill, Rational materials design of sorbent coatings for explosives: applications with chemical sensors, Talanta 54 (2001) 469-485.

[6] P. Lewis, R. Manginell, D. Adkins, R. Kottenstette, D.R. Wheeler, S. Sokolowski, D. Trudell, J. Byrnes, M. Okandan, J. Bauer, R. Manley, C. Frye-Mason, Recent advancements in the gas-phase microchemlab, IEEE Sens. J. 6 (2006) 784-795.

[7] M.S. Meaney, V.L. McGuffin, Luminescence-based methods for sensing and detection of explosives, Anal. Bioanal. Chem. 391 (2008) 2557-2576.

[8] J.S. Yang, T.M. Swager, Fluorescent porous polymer films as tnt chemosensors: electronic and structural effects, J. Am. Chem. Soc. 120 (1998) 11864-11873. 
[9] W.C. Trogler, M.J. Sailor, Detection of nitrobenzene, dnt, and tnt vapors by quenching of porous silicon photoluminescence, Chem. Eur. J. 6 (2000) 2205-2213.

[10] J. Zhang, X. Tang, J. Dong, T. Wei, H. Xiao, Zeolite thin film coated long period fiber grating sensor for measuring trace chemical, Opt. Express 16 (2008) 8317-8323.

[11] S.M. Daly, M. Grassi, D.K. Shenoy, F. Ugozzoli, E. Dalcanale, Supramolecular surface plasmon resonance (spr) sensors for organophosphorus vapor detection, J. Mater. Chem. 17 (2007) 1809-1818.

[12] A. Rose, Z. Zhu, C.F. Madigan, T.M. Swager, V. Bulovic, Sensitivity gains in chemosensing by lasing action in organic polymers, Nature 434 (2005) 876-879.

[13] L. Pinnaduwage, A. Wig, D. Hedden, A. Gehl, D. Yi, T. Thundat, R. Lareau, Detection of trinitrotoluene via deflagration on a microcantilever, J. Appl. Phys. 95 (2004) 5871-5875.

[14] C.W. Van Neste, L.R. Senesac, D.Yi, T. Thundat, Standoff detection of explosive residues using photothermal microcantilevers, Appl. Phys. Lett. 92 (2008) 134102.

[15] G. Bunte, J. Hurttlen, H. Pontius, K. Hartlieb, H. Krause, Gas phase detection of explosives such as 2,4,6-trinitrotoluene by molecularly imprinted polymers, Anal. Chim. Acta $591(2007) 49-56$.

[16] Y. Tomita, M.H. Ho, G.G. Guilbault, Detection of explosives with a coated piezoelectric quartz crystal, Anal. Chem. 51 (1979) 1475-1478.

[17] W.J. Buttner, M. Findlay, W. Vickers, W.M. Davis, E.R. Cespedes, S. Cooper, J.W. Adams, In situ detection of trinitrotoluene and other nitrated explosives in soils, Anal. Chim. Acta $341(1997) 63-71$.

[18] J. Wang, Electrochemical sensing of explosives, Electroanal. 19 (2007) 415-423. 
[19] R.A. Munoz, D. Lu, A. Cagan, J. Wang, One-stepsimplified electrochemical sensing of tatp based on its acid treatment, Analyst 132 (2007) 560-565.

[20] M. Krausa, K. Schorb, Trace detection of 2,4,6-trinitrotoluene in the gaseous phase by cyclic voltammetry, J. Electroanal. Chem. 461 (1999) 10-13.

[21] Y. Sun, J. Liu, G. Frye-Mason, S.j. Ja, A.K. Thompson, X. Fan, Optofluidic ring resonator sensors for rapid dnt vapor detection, Analyst 134 (2009) 1386-1391.

[22] A. Pettersson, S. Wallin, H. Ostmark, A. Ehlerding, I. Johansson, M. Nordberg, H. Ellis, A. Al-Khalili, Explosives standoff detection using Raman spectroscopy: from bulk towards trace detection, Proc. SPIE 7664 (2010) 76641K-76641K-12.

[23] J. Kneipp, H. Kneipp, K. Kneipp, SERS—a single-molecule and nanoscale tool for bioanalytics, Chem. Soc. Rev. 37 (2008) 1052-1060.

[24] D.L. Jeanmaire, R.P. Van Duyne, Surface raman spectroelectrochemistry: Part i. heterocyclic, aromatic, and aliphatic amines adsorbed on the anodized silver electrode, J. Electroanal. Chem. Interfacial. Electrochem. 84 (1997) 1-20.

[25] M. Fleischmann, P. Hendra, A. McQuillan, Raman spectra of pyridine adsorbed at a silver electrode, Chem. Phys. Lett. 26 (1974) 163-166.

[26] G.L. Liu, L.P. Lee, Nanowell surface enhanced Raman scattering arrays fabricated by softlithography for label-free biomolecular detections in integrated microfluidics, Appl. Phys. Lett. 87 (2005) 074101.

[27] C.J. Choi, Z. Xu, H.Y. Wu, G.L. Liu, B.T. Cunningham, Surface-enhanced Raman nanodomes, Nanotechnology 21 (2010) 415301.

[28] M.G. Albrecht, J.A. Creighton, Anomalously intense raman spectra of pyridine at a silver electrode, J. Am. Chem. Soc. 99 (1977) 5215-5217. 
[29] F. Hao, C.L. Nehl, J.H. Hafner, P. Nordlander, Plasmon resonances of a gold nanostar, Nano Lett. 7 (2007), 729-732.

[30] F. Yan, M.B. Wabuyele, G.D. Griffin, A.A. Vass, T. Vo-Dinh, Surface-enhanced raman scattering detection of chemical and biological agent simulants, IEEE Sens. J. 5 (2005) 665-670.

[31] B. Mondal, S.K. Saha, Fabrication of SERS substrate using nanoporous anodic alumina template decorated by silver nanoparticles, Chem. Phys. Lett. 497 (2010) 89-93.

[32] A. Gutes, C. Carraro, R. Maboudian, Silver dendrites from galvanic displacement on commercial aluminum foil as an effective SERS substrate, J. Am. Chem. Soc. 132 (2010) 1476-1477.

[33] B.B. Xu, Y.L. Zhang, W.Y. Zhang, X.Q. Liu, J.N. Wang, X.L. Zhang, D.D. Zhang, H.B. Jiang, R. Zhang, H.B. Sun, Silver-Coated Rose Petal: Green, Facile, Low-Cost and Sustainable Fabrication of a SERS Substrate with Unique Super hydrophobicity and High Efficiency, Adv. Opt. Mater. 1 (2013) 56-60.

[34] C. Cheng, B. Yan, S.M. Wong, X. Li, W. Zhou, T. Yu, S. Zexiang, Y. Hongyu, H.J. Fan, Fabrication and SERS performance of silver-nanoparticle-decorated $\mathrm{Si} / \mathrm{ZnO}$ nanotrees in ordered arrays, ACS Appl. Mater. Interfaces 2 (2010) 1824-1828.

[35] G.F. Andrade, M. Fan, A.G. Brolo, Multilayer silver nanoparticles-modified optical fiber tip for high performance SERS remote sensing, Biosens. Bioelectron. 25 (2010) 2270-2275.

[36] C.H. Lee, L. Tian, and S. Singamaneni, Paper-based SERS swab for rapid trace detection on real-world surfaces, ACS Appl. Mater. Interfaces 2 (2010) 3429-3435. 
[37] A.K. Misra, S.K. Sharma, L. Kamemoto, P.V. Zinin, Q. Yu, N. Hu, L. Melnick, Novel micro-cavity substrates for improving the raman signal from submicrometer size materials, Appl. Spectrosc. 63 (2009) 373-377.

[38] J.D. Driskell, S. Shanmukh, Y. Liu, S.B. Chaney, X.J. Tang, Y.P. Zhao, R.A. Dluhy, The use of aligned silver nanorod arrays prepared by oblique angle deposition as surface enhanced Raman scattering substrates, J. Phys. Chem. C 112 (2008) 895-901.

[39] E.C. Le Ru, P.G. Etchegoin, J. Grand, N. Felidj, J. Aubard, G. Levi, A. Hohenau, J.R. Krenn, Surface enhanced Raman spectroscopy on nanolithography-prepared substrates, Curr. Appl. Phys. 8 (2008) 467-470.

[40] E.D. Diebold, N.H. Mack, S.K. Doorn, E. Mazur, Femtosecond laser-nanostructured substrates for surface-enhanced Raman scattering, Langmuir 25 (2009) 1790-1794.

[41] M. Hu, J. Tang, F.S. Ou, H.P. Kuo, S.Y. Wang, Z. Li, R.S. Williams, Metal-coated Si nanograss as highly sensitive SERS sensors, Proc. SPIE (2009) 73120I-73120I.

[42] A. Eshkeiti, B.B. Narakathu, A.S.G. Reddy, A. Moorthi, M.Z. Atashbar, E. Rebrosova, M. Rebros, M. Joyce. Detection of heavy metal compounds using a novel inkjet printed surface enhanced Raman spectroscopy (SERS) substrate. Sens. Actuator B-Chem. $171(2012)$ 705-711.

[43] A. Eshkeiti, M. Rezaei, B.B. Narakathu, A.S.G. Reddy, S. Emamian, M.Z. Atashbar, Gravure printed paper based substrate for detection of heavy metals using surface enhanced Raman spectroscopy (SERS), IEEE Sens. (2013) 1-4.

[44] M.F.J. Bohan, T.C. Claypole, D.T. Gethin, The effect of process parameters on product quality of rotogravure printing, Proc. Inst. Mech. Eng., B J. Eng. Manuf. 214 (2000) 205219. 
[45] A.G. Brolo, D.E. Irish, G. Szymanski, J. Lipkowski, Relationship between SERS intensity and both surface coverage and morphology for pyrazine adsorbed on a polycrystalline gold electrode, Langmuir 14 (1998) 517-527.

[46] E.L. Holthoff, D.N. Stratis-Cullum, M.E. Hankus, A nanosensor for TNT detection based on molecularly imprinted polymers and surface enhanced Raman scattering, Sensors $11(2011)$ 2700-2714.

[47] A. Gopinath, S.V. Boriskina, B.M. Reinhard, L.D. Negro, Deterministic aperiodic arrays of metal nanoparticles for surface-enhanced Raman scattering (SERS), Opt. Express 17 (2009) 3741-3753.

[48] L. Gunnarsson, T. Rindzevicius, J. Prikulis, B. Kasemo, M. Kall, S. Zou, G.C. Shatz, Confined plasmons in nanofabricated single silver particle pairs: experimental observations of strong interparticle interactions, J. Phys. Chem. B 109 (2005) 1079-1087.

[49] S. Xie, E. Alexis, T. Bell, Effects of temperature on the Raman spectra and dispersed oxides, J. Phys. Chem. B 22 (2001) 5144-5152.

[50] M.Z. Atashbar, S. Singamaneni, Comparative studies of temperature dependence of G-band peak in single walled carbon nanotube and highly oriented pyrolytic graphite, Appl. Phys. Lett. 86 (2005) 123112. 


\section{Figures Captions}

Fig 1. A schematic illustration of SERS "hotspots" between adjacent silver nanoparticles. These hotspots generate strong electromagnetic fields that cause the enhancement of Raman scattering. The average size for the silver nanoparticle is $150 \mathrm{~nm}$.

Fig 2. The schematic of gravure printing process.

Fig 3. Photograph of a $1 \mathrm{~cm} \times 2 \mathrm{~cm}$ SERS substrate fabricated by gravure printing silver nanoparticles on a PET sheet and cured at $130{ }^{\circ} \mathrm{C}$ for 20 minutes in an oven.

Fig 4. Measurement of the thickness and roughness for the gravure printed silver nanoparticle ink on PET using 3D images obtained from the Bruker vertical scanning interferometer microscope (CounterGT) with 50X magnification: (a) thickness of $900 \mathrm{~nm}$ along the Y profile and (b) RMS roughness of $150 \mathrm{~nm}$.

Fig 5. Experiment setup for transfer of DNT vapor onto SERS substrate. A steel wool, exposed to $1 \mathrm{ml}$ of the DNT saturated solution, was inserted into a heated copper pipe and nitrogen gas was used to blow the DNT vapor onto the SERS substrate.

Fig 6. Experiment setup for measurement of SERS response of gravure printed substrate.

Fig 7. Comparison of Raman spectra obtained for DNT (a) solution and (b) vapor on bare PET (green) versus printed SERS substrate (blue) using $785 \mathrm{~nm}$ excitation. An enhancement factor of three and four for the peaks at $1350.13 \mathrm{~cm}^{-1}$ was observed for the SERS based response of the printed substrate towards DNT solution and vapor, respectively, when compared to target molecules adsorbed on bare PET.

Fig 8. Raman spectra of DNT vapor on SERS substrate heated from $25{ }^{\circ} \mathrm{C}$ to $65^{\circ} \mathrm{C}$, in steps of $10{ }^{\circ} \mathrm{C}$ using a hot plate. Results show $85 \%$ decrement in the intensity of the Raman spectra, for the peaks at $1350.13 \mathrm{~cm}^{-1}$, as temperature was increased.

Fig 9. SERS response of DNT vapor on Ag NP film at different bending angles. Intensity of the Raman spectrum was increased by $100 \%, 200 \%$, and $300 \%$ as the substrate was bended to different radius of curvatures $(3.4 \mathrm{~cm}, 2.1 \mathrm{~cm}$ and $1.6 \mathrm{~cm})$. 


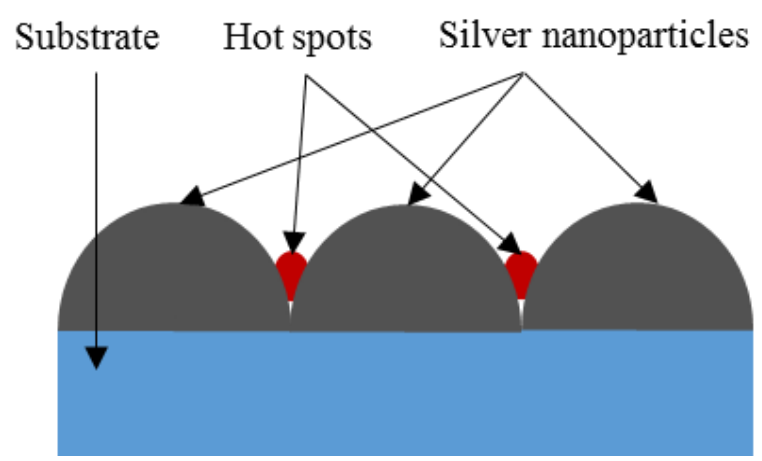

Figure 1 


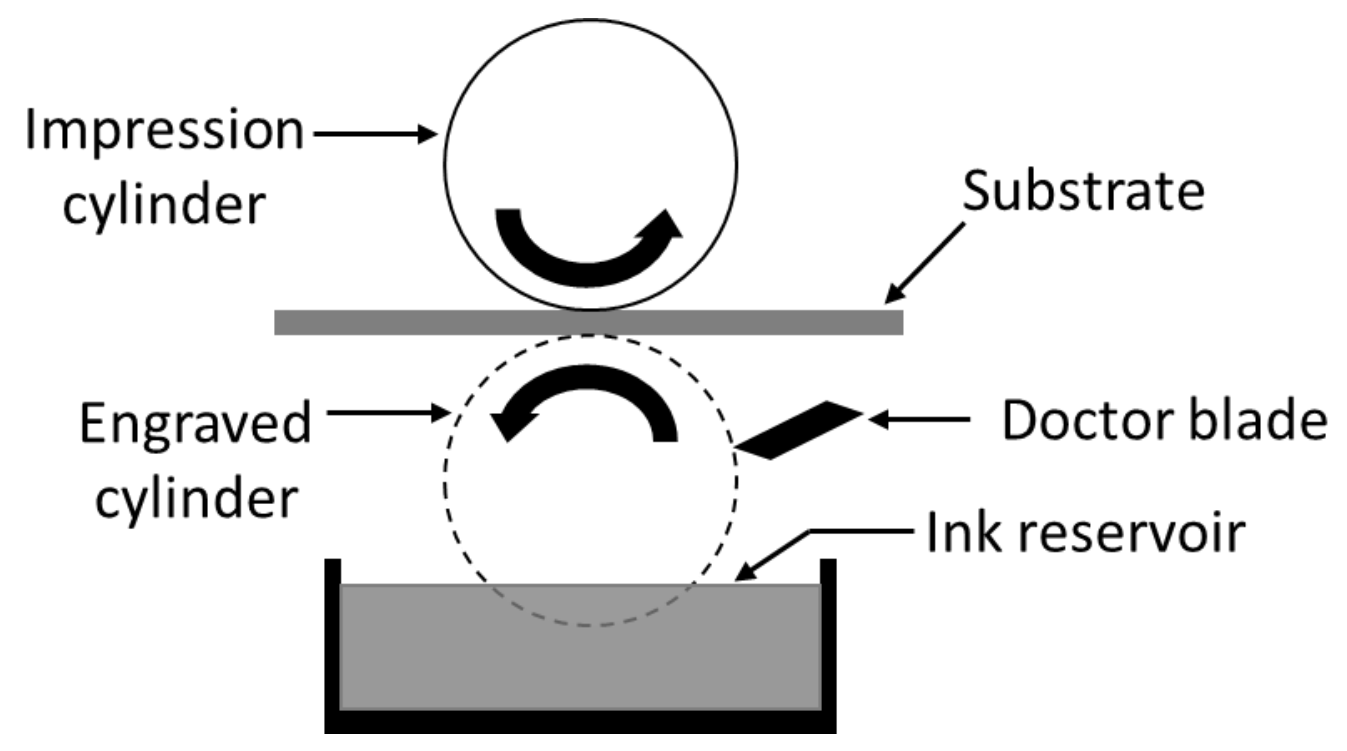

Figure 2 


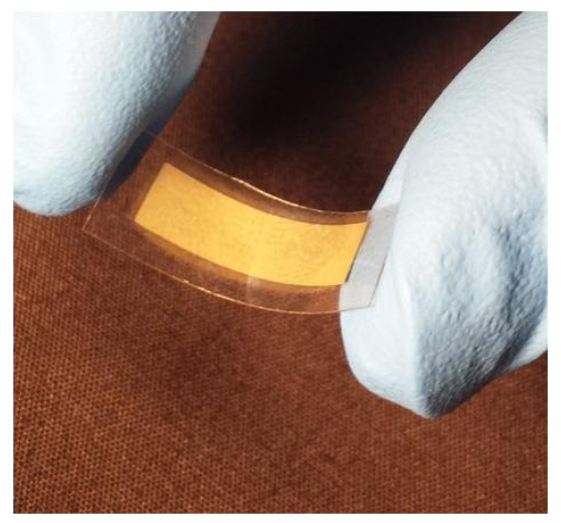

Figure 3

Page 20 of 28 

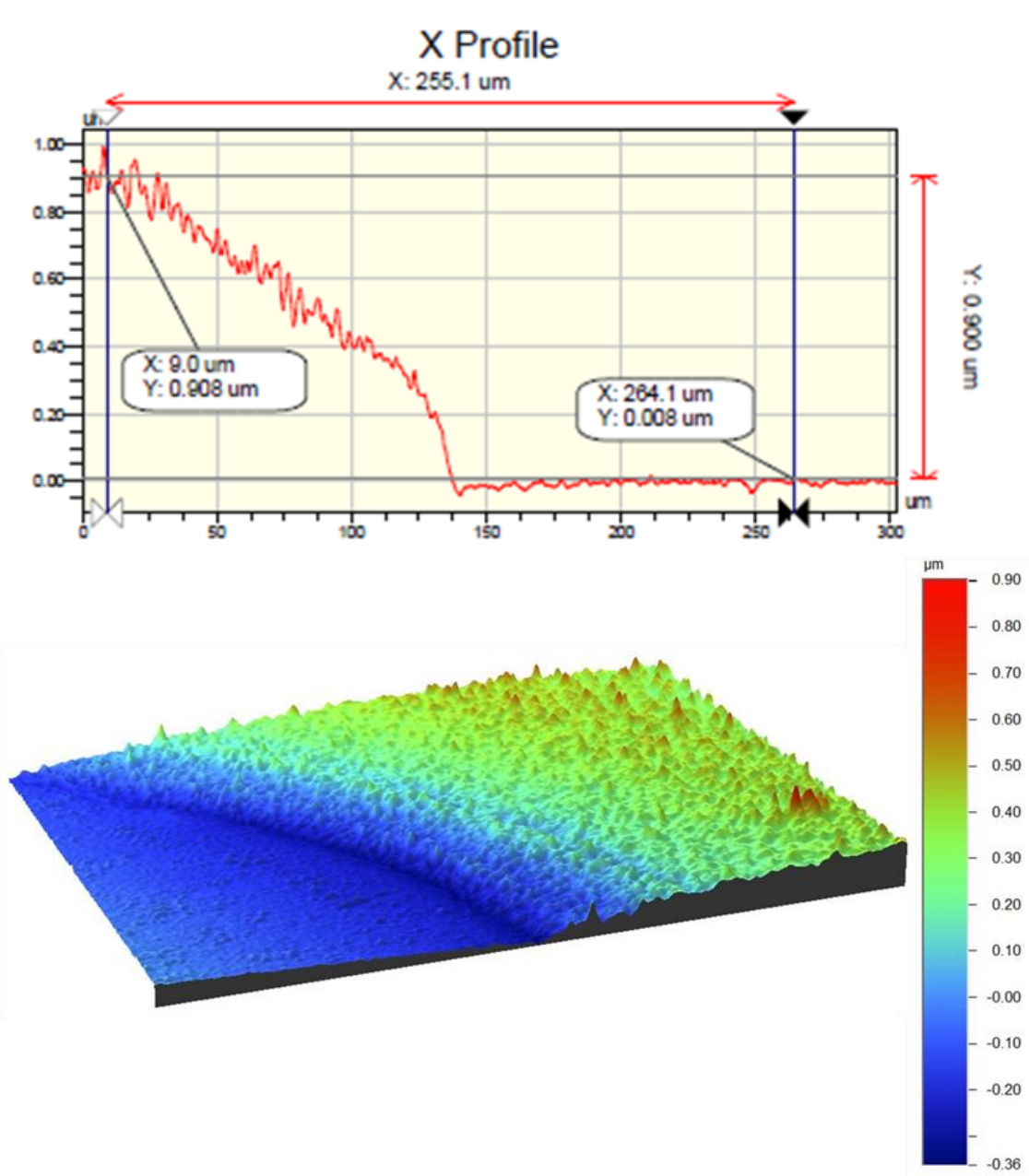

(a)

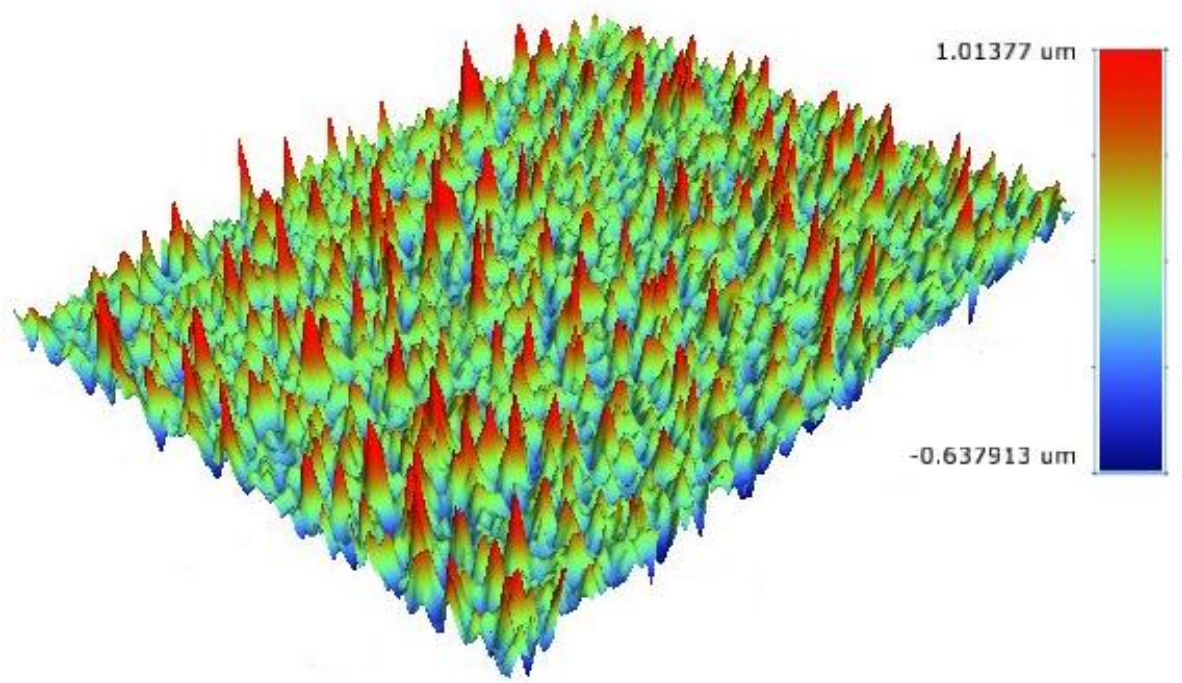

Analytical Results
\begin{tabular}{|l|l|l|}
\hline Label & Value & Units \\
\hline Average & -0.011 & $\mu \mathrm{m}$ \\
\hline Data Points & 15876 & \\
\hline Percent Data Points & 5.17 & $\%$ \\
\hline$R a$ & 0.085 & $\mu \mathrm{m}$ \\
\hline$R p$ & 0.797 & $\mu \mathrm{m}$ \\
\hline$R q$ & 0.112 & $\mu \mathrm{m}$ \\
\hline$R t$ & 1.148 & $\mu \mathrm{m}$ \\
\hline$R v$ & -0.35 & $\mu \mathrm{m}$ \\
\hline
\end{tabular}

(b)

Figure 4 


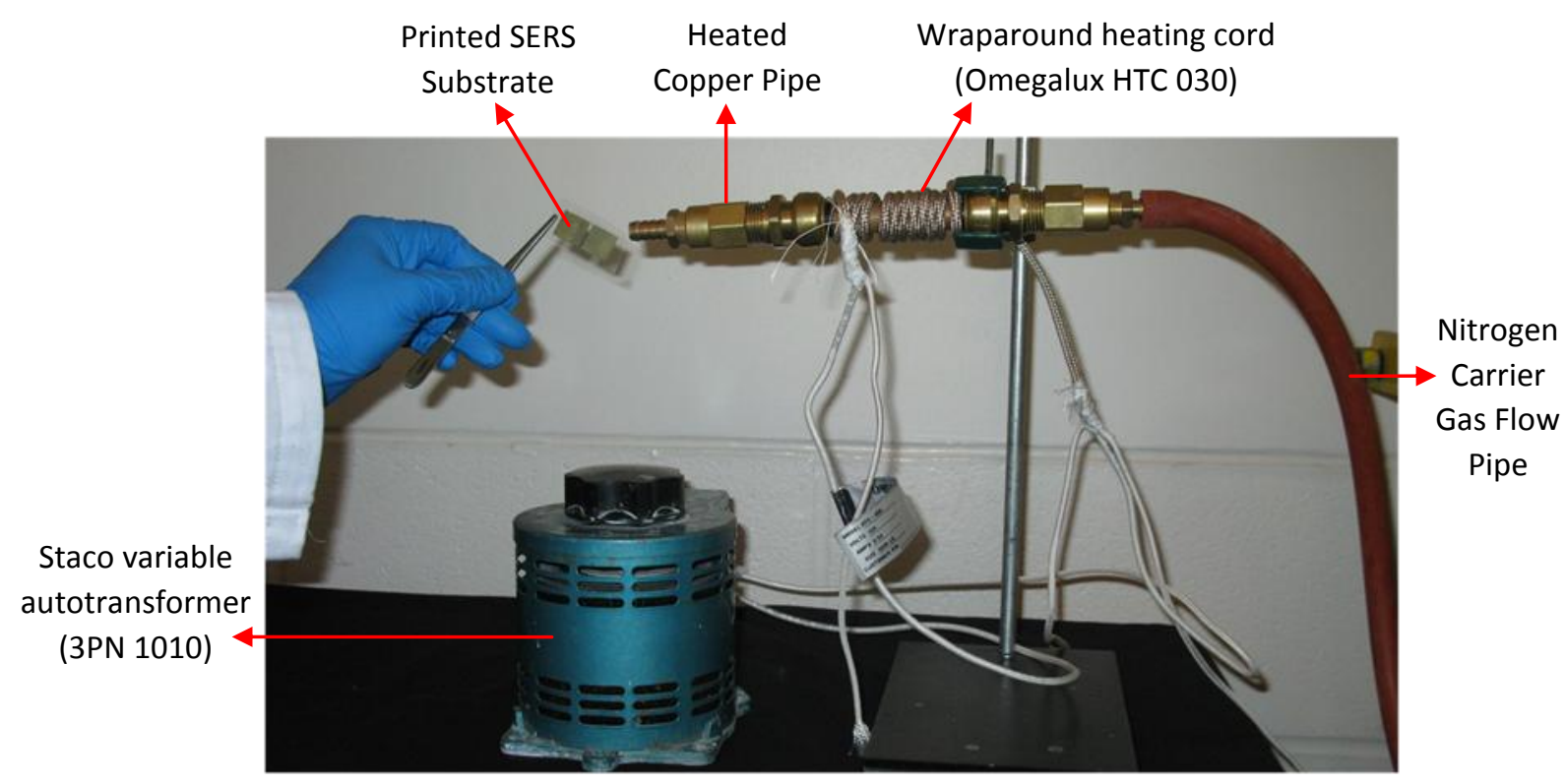

Figure 5 


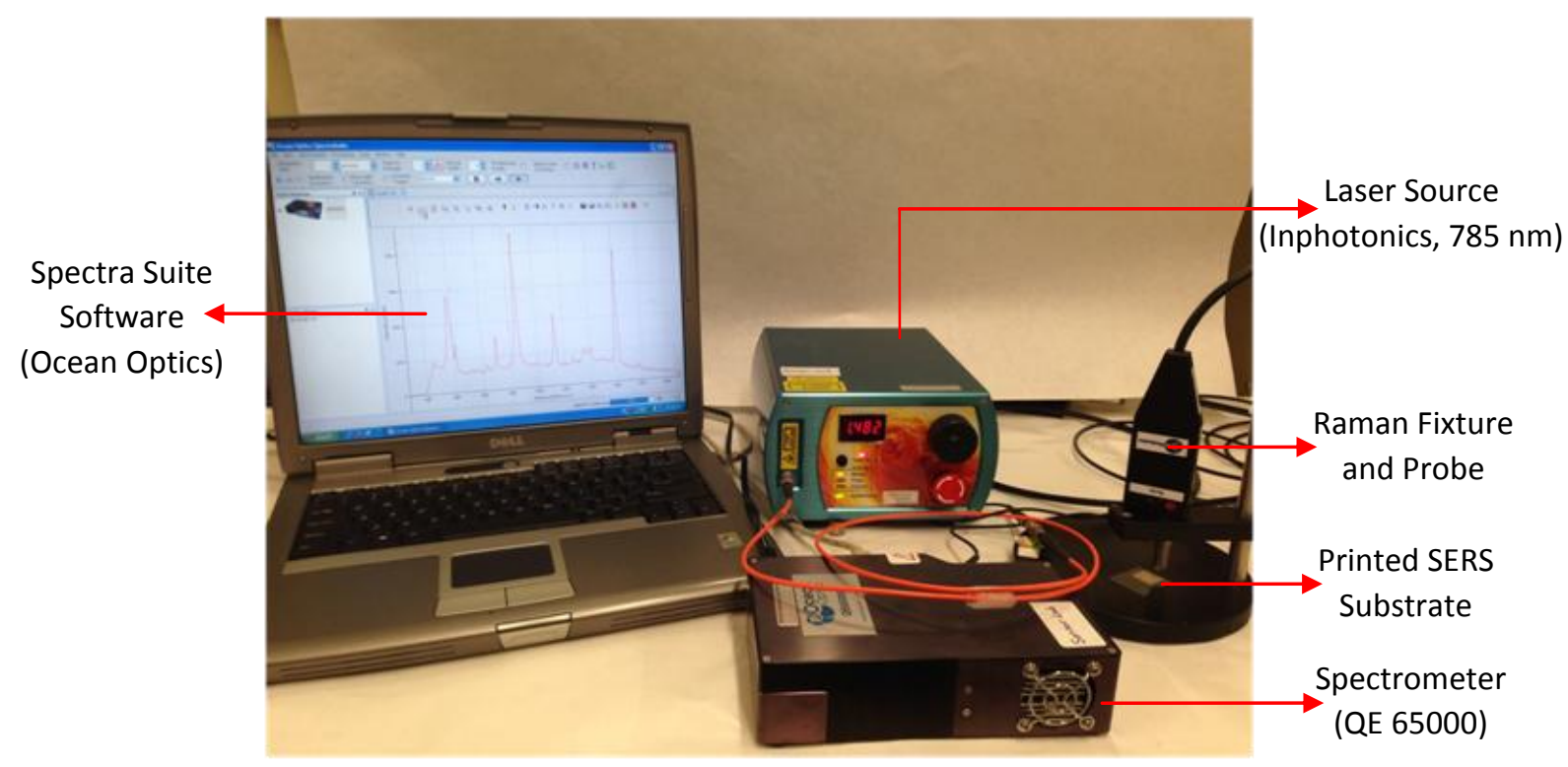

Figure 6 


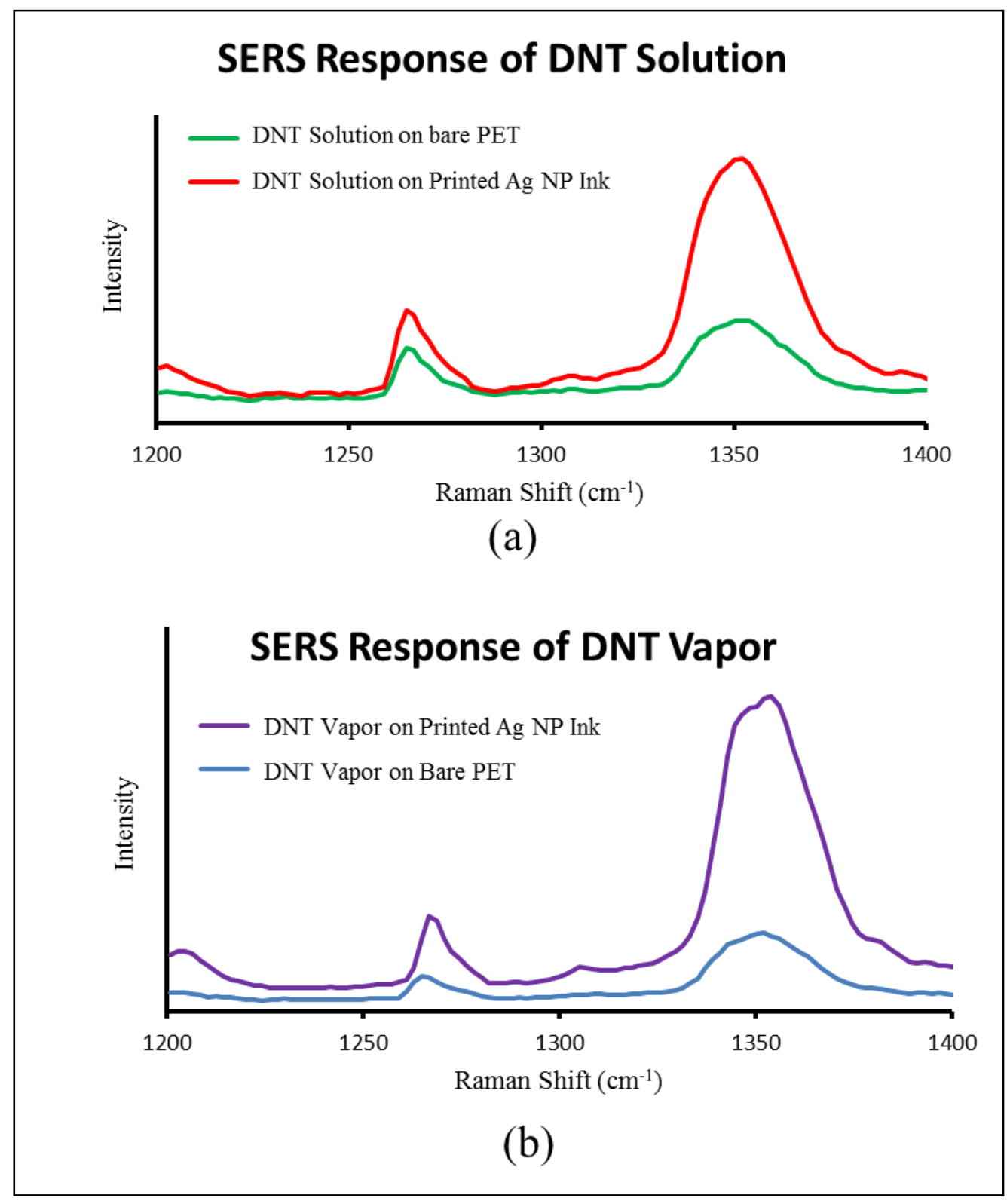

Figure 7 


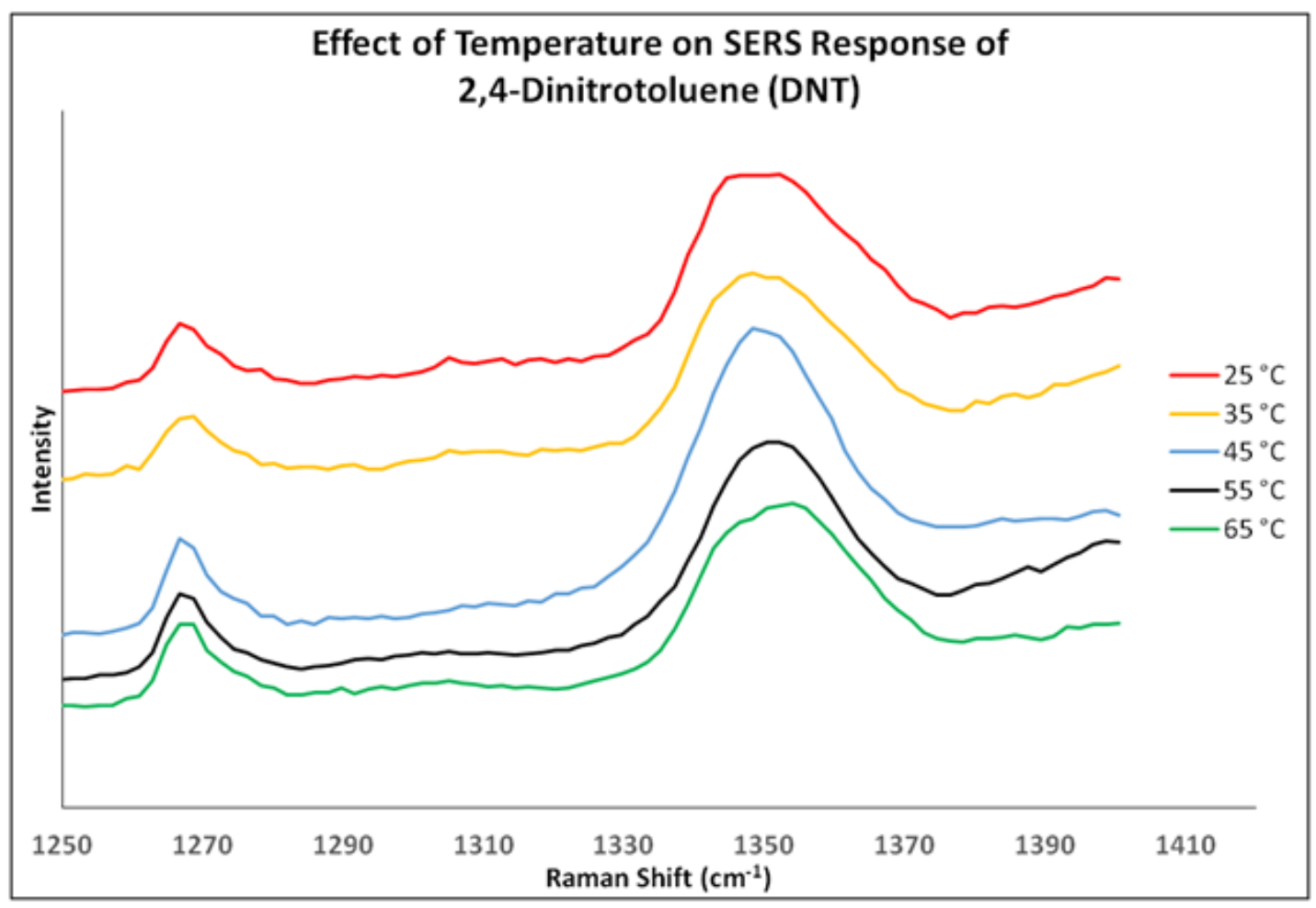

Figure 8 


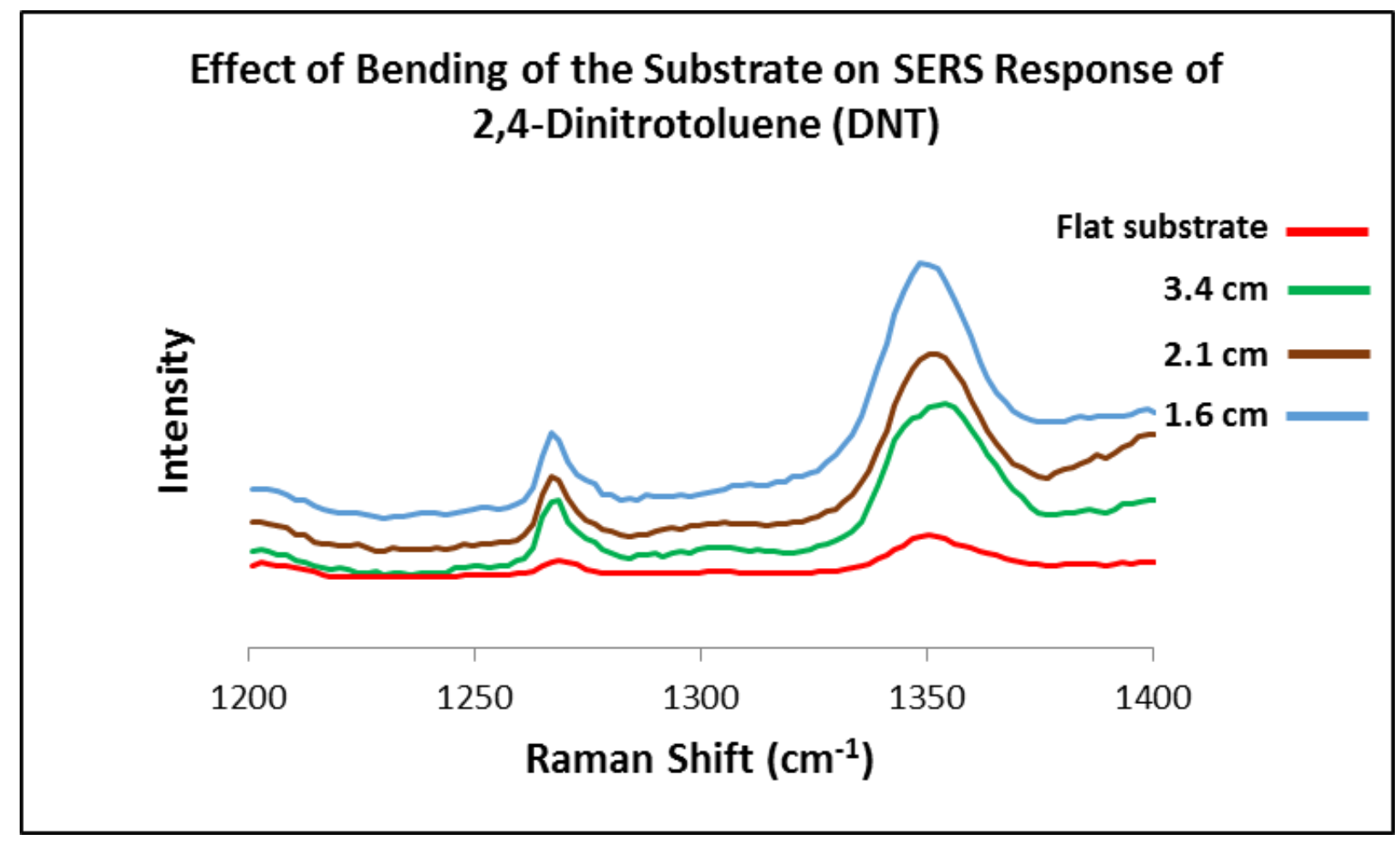

Figure 9 


\section{Author Biographies}

Sepehr Emamian received the B.Sc. degree in electrical engineering from the Isfahan University of Technology, Isfahan, Iran, the M.Sc. degree in electrical engineering from the Sharif University of Technology, Tehran, Iran. He is currently pursuing the Ph.D. degree in Electrical and Computer Engineering at Western Michigan University, Kalamazoo, USA. His research interest includes control systems and design, fabrication, and characterization of printed sensor structures, Schottky diodes, transistors, biochemical sensing systems, solar cells, energy storage devices and wearable sensors.

Ali Eshkeiti received the B.Sc. degree in Electronics Engineering from Azad University, Tehran Iran and the M. Sc. degree in Electrical Engineering at Western Michigan University, Kalamazoo, USA. He is currently pursuing the Ph.D. degree in Electrical and Computer Engineering at Western Michigan University, Kalamazoo, USA. His research interest includes design, fabrication, and characterization of printed sensor structures, transistors, biochemical sensing systems, solar cells, energy storage devices and lab-on-a-chip sensing systems.

Binu Baby Narakathu received the B.E. degree in Electronics and Communication from Visvesvaraya Technological University, Bangalore, India and the M.Sc. degree in Computer Engineering from Western Michigan University, Kalamazoo, USA in 2009. He is currently pursuing Ph.D. in the Department of Electrical and Computer Engineering at Western Michigan University, Kalamazoo, USA. His research interests include all aspects of design, fabrication, and characterization of high performance sensing systems, microfluidic devices, lab-on-a-chip for point-of-care testing (POCT), biosensors, bioelectronics, printed electronic devices and BioMEMS devices for applications in the biomedical, environmental and defense industries. 
Sai Guruva Reddy Avuthu received the B.Tech. degree in Electronics and Computer Engineering from Jawaharlal Nehru Technological University, Hyderabad, AP, India and the M.Sc. degree in Electrical Engineering from Western Michigan University, Kalamazoo, USA in 2011. He is currently pursuing the Ph.D. degree in the Department of Electrical and Computer Engineering at Western Michigan University, Kalamazoo, USA. His research interests include design, fabrication, and characterization of printed sensor structures, transistors, microfluidics and lab-on-a-chip sensing systems.

Dr. Massood Z. Atashbar received the B.Sc. degree in Electrical Engineering from the Isfahan University of Technology, Isfahan, Iran, the M.Sc. degree in Electrical Engineering from the Sharif University of Technology, Tehran, Iran, and the Ph.D. degree from the Department of Communication and Electronic Engineering, Royal Melbourne Institute of Technology University, Melbourne, Australia, in 1998. From 1998 to 1999, he was a Postdoctoral Fellow with the Center for Electronic Engineering and Acoustic Materials, The Pennsylvania State University, University Park. He is currently a Professor with the Electrical and Computer Engineering Department, Western Michigan University, Kalamazoo. His research interests include physical and chemical microsensor development, wireless sensors, and applications of nanotechnology in sensors, digital electronics, and printed electronic devices. He is the author of more than 160 articles in refereed journals and refereed conference proceedings. He is a member of the editorial board and associate editor for the journal of IEEE Sensors. 